Alonso Amezua, I., Azpeitia Eizagirre, A. Iriondo Arana, I. \& Zulaika Galdos, T. (2017). Autoconfrontación a la propia actividad de enseñanza. Formación e investigación de la formación. Revista Electrónica Interuniversitaria de Formación del Profesorado, 20(2), 169-182.

DOI: http://dx.doi.org/10.6018/reifop.20.1.237261

\title{
Autoconfrontación a la propia actividad de enseñanza. Formación e investigación de la formación
}

Idurre Alonso Amezua', Agurtzane Azpeitia Eizagirre1, Itziar Iriondo Arana², Teresa Zulaika Galdos $^{2}$

${ }^{(1)}$ Mondragon Unibertsitatea, ${ }^{(2)}$ Universidad del País Vasco UPV/EHU

\section{Resumen}

Partimos de un posicionamiento epistemológico accional, praxiológico que toma la actividad humana (aquí la enseñanza de las lenguas y la formación) en tanto objeto de estudio. La formación inicial del profesorado se aborda en tanto proceso en devenir, en relación con dispositivos de formación que se conciben y examinan con vistas a favorecer el desarrollo de la acción de los formados. Los elementos teóricos convocados provienen de diversos modelos de la acción y de la actividad humana, en particular la teoría de la acción social.

Los métodos de investigación y de formación que se emplean tienen como finalidad la producción de trazas de la actividad, al tiempo que proporcionan espacios adecuados para su análisis por los actores. Las grabaciones - principalmente en vídeo - se utilizan para realizar entrevistas de autoconfrontación que son a su vez momentos propicios para la producción de significados en relación con las actividades observadas.

Los primeros resultados obtenidos muestran algunas de las actividades típicas realizadas por estudiantes durante el Practicum en relación con la enseñanza de lenguas y los fenómenos

\section{Contacto:}

Idurre Alonso Amezua, ialonso@,mondragon.edu, Mondragon Unibertsitatea, Dorleta 20540 Eskoriatza. Esta investigación es parte del proyecto EDU2012-37889. Asimismo, el análisis del corpus que se muestra ha sido facilitado por la Red HIPREST (asociada a la Cátedra de la Unesco: Former les enseignants au XXlème s.) de la que somos miembro. La Red está conformada por la Universidad del Pais Vasco y Mondragon Unibertsitatea. 
formativos producidos. Estos resultados son empleados a su vez para acondicionar los entornos de formación basados en el análisis de la actividad docente.

\section{Palabras clave}

Formación inicial del profesorado; video formación; actividad humana; tipificación.

\section{Self-confrontation of own teaching activity. Training and research on training}

\section{Abstract}

The starting point of this work is an epistemological, actional and praxiological position that adopts human activity (language teaching and training, in this case) as the object of study. Teachers' initial training is dealt with as a developing process related to training devices conceived and analysed with the aim of enabling the development of trainees' action. The assembled theoretical elements result from different models of action and human activity, especially from the theory of social action (Schütz, 1950).

Research and training methods that have been used aim at generating traces of activity as long as providing appropriate opportunities for agents to analyse them. Recordings -especially video-recordings- are used for self-confrontation interviews that supply adequate instances to produce meanings related to observed activities.

The first results show some of the typical activities in language learning that students have carried out during their Practicum period and the generated training phenomena. Those results are, subsequently, used to set up training-environments based on the analysis of teachingactivity.

\section{Key words}

Teacher training; video training; novice teachers; typification.

\section{Introducción}

En este artículo presentamos el trabajo de formación y de investigación que realiza la red HIPREST en el País Vasco, trabajo que se caracteriza primeramente por su doble objetivo de formación de profesorado y de investigación de la formación. De esta manera tratamos de superar una visión aplicacionista de la formación del profesorado, que tomaría los resultados de la investigación en campos tales como la pedagogía, la didáctica o las ciencias de referencia (psicología, lingüística, matemática, etc.) como únicas fuentes para la elaboración de contenidos de formación. En ese sentido, es significativo que los profesores noveles, tanto en 
nuestro entorno como en el panorama internacional se muestren preocupados por la distancia que existe entre sus conocimientos y la actividad real (Arocena y Gorter, 2013).

Tal y como señalan los autores Mir y Ferrer (2014), hay un vacío en la formación inicial de los docentes para afrontar los cambios y las circunstancias a los que el hecho educativo se ve sometido en la actualidad. Es por ello que en países como el Reino Unido, Francia o Suiza empiezan a abordar los procesos de formación inicial "en el lugar de trabajo". A su vez, varias propuestas emergentes tanto en el Estado español como en los países citados se basan en el principio mencionado por Alsina (2013), según el cual el conocimiento sobre la práctica docente debe ser un conocimiento creado por el mismo sujeto en formación y no un conocimiento ya creado con anterioridad por terceros y transmitido por ellos.

Consideramos, además, que, en los procesos de formación, junto con la del profesor experto, debe tomarse en cuenta la actividad del profesor novel, en tanto objeto de conocimiento, con sus particularidades, sus intentos, sus dificultades y su potencialidad. La investigación de la formación que considera ésta como proceso deberá, pues, interesarse en las etapas que constituyen el inicio de una adquisición progresiva del oficio centrándose en las actividades típicas que contribuyen a la enseñanza de lenguas en contextos plurilingües. Estos objetivos de conocimiento llevan a adoptar marcos teóricos, conceptos y metodologías que focalizan en la actividad humana.

Queremos subrayar que en nuestra orientación la metodología reviste gran importancia, ya que juega un doble papel: está al servicio del análisis de la actividad y al mismo tiempo entra a formar parte del entorno de formación.

\section{Orientación teórica y metodológica}

\section{La actividad y la experiencia del actor}

Este trabajo de investigación se apoya teóricamente en diversos modelos de la acción o actividad humana, entre los cuales cabe destacar la teoría de la acción social de A. Schütz, el pragmatismo de Dewey (1933), las bases enunciativas y de análisis discursivo del interaccionismo socio-discursivo de Bronckart (1997), y la actividad del lenguaje de la escuela rusa de filosofía y psicología de Bajtín (1982) y Voloshinov (1992).

Uno de los puntos de partida del posicionamiento teórico en el que nos apoyamos es el siguiente: para comprender "la actividad humana" es necesario profundizar en el significado que lo realizado posee para el actor, es decir, en su experiencia. La teoría de la acción social que Schütz desarrolla en 1932 partiendo de los trabajos de Husserl, aunque se distancie de ellos, trata precisamente del modo de aprehensión de la experiencia y del modo de construcción de contextos de significado (Schütz, 1993). Ciertos aspectos de estas teorías son clave para nuestras investigaciones. Trataremos de presentarlos en las siguientes líneas.

Se postula que el actor percibe las situaciones de acción a través de tipos. Los tipos toman la forma de creencias, de modos de percepción transmitidos por el entorno social (familiar, 
escolar, formativo, etc.) y apuntan hacia los modos típicos de resolver situaciones típicas de acción, por aplicación de métodos o de recetas típicas (Cefaï, 1994). Estos tipos se elaboran socialmente y están organizados culturalmente. El conocimiento del actor deriva de estas construcciones sociales que lo preceden $y$ que se transmiten entre los actores, fundamentalmente por medio del lenguaje común.

Ante una situación concreta, el actor opera gracias a un proceso de reflexión (Schütz emplea este término en el sentido de reflejo, no de operación intelectual), es decir, realiza una vuelta hacia el pasado que da lugar a una síntesis de identificación y de reconocimiento que responde a la cuestión de saber de qué situación de acción se trata, a qué otra situación similar recuerda, etc. Dicha reflexión se basa en los tipos disponibles para el actor, en sus "reservas de experiencia". Pero los modos típicos de actuar no son retomados de manera mecánica. En la vida cotidiana el actor moviliza "esquemas de experiencia" para aprehender la tipicidad ya presente o para tipificar configuraciones fenomenológicas novedosas, inéditas o desconocidas. Los esquemas están "encarnados" en las actividades prácticas y en las actividades interpretativas. Las tipificaciones que el actor realiza ante toda situación de acción no corresponden a la lógica formal, no se trata de operaciones de conocimiento abstracto, ni se basan en generalizaciones que parten de observaciones concretas (en oposición a otras teorías de los tipos, tales como la de Rosch, 1978). Se trata, al contrario, de un transfer perceptivo al campo práctico-sensible, de un "ver-como" instantáneo.

De lo expuesto brevemente retenemos la idea de que la experiencia antepredicativa del actor está organizada en tipos de actividad, que son sociales; el actor recurre a ellos y los utiliza en sus experiencias en un ejercicio incesante de tipificación. La rememoración de las actividades ya realizadas permite "re-tomarlas" a la hora de aprehender una nueva situación y actuar en ella. Por otro lado, ello va acompañado de anticipaciones de la experiencia que va a realizarse.

Este posicionamiento teórico subraya la importancia que, a la hora de actuar, tienen las actividades ya realizadas por el actor, junto con sus contextos de significación. El posicionamiento se interesa, por otro lado, por los modos corporales, es decir "encarnados", no exclusivamente intelectuales, de integrar la actividad por parte del actor. De acuerdo, tanto con la orientación de la cognición-acción situada (Suchmann, 1987), como con la de la ergonomía cognitiva (De Montmollin, 1986; Salembier, Theureau, Zouinar y Vermersch, 2001), se critica el modelo racional del actor según el cual éste opera siguiendo un plan, por ejemplo, una prescripción en el trabajo. Por otro lado, la actividad se concibe como multiforme: el actor moviliza las formas que revisten sus experiencias anteriores y si es necesario, retoma aquellos recursos ya utilizados adecuándolos a la nueva situación; se refiere a formas normalizadas, a otras formas que ha podido observar, a variantes hipotéticas, etc. Por lo tanto, la actividad concreta convoca múltiples formas de acción y, en particular, las formas elaboradas socialmente. 


\section{Metodología de análisis de la actividad}

Los métodos que proponemos para la investigación y la formación tienen como finalidad, primeramente, producir trazas de la actividad y a continuación, proporcionar espacios adecuados para su análisis por parte del actor. Este marco metodológico se enraíza en la ergonomía cognitiva francófona (Clot, Faïta, Fernandez y Scheller, 2001; Durand, 2008; Theureau, 2010) y conoce un gran desarrollo en el ámbito francófono de la formación del profesorado (Ria y Leblanc, 2011; Lussi Borer, Muller, Ria, Saussez y Vidal-Gomel,2014).

La utilización del vídeo como instrumento de formación y desarrollo profesional del profesorado es una práctica cada vez más extendida aunque sus resultados no se hayan reflejado de una manera sistemática en estudios de ámbito internacional (Seidel et al., 2011). Existen algunas investigaciones al respecto, especialmente en el mundo francófono (Gaudin y Chaliès, 2012 ; Lussi Borer et al., 2014) así como en el ámbito más específico de la enseñanza de las matemáticas, principalmente, de los Estados Unidos (Santagata y Yeh, 2015; Sherin, 2001 ; van Es y Sherin, 2008) no obstante, aún habría que precisar cómo aprenden los docentes en entornos de video-formación.

En este epígrafe presentamos el dispositivo metodológico empleado en la investigación. Dicha investigación comprende tres etapas.

1. La formación universitaria del profesor novel. El alumnado recibe formación en metodologías basadas en el análisis de la actividad a través de seminarios impartidos por sus tutoras de la Universidad. En ellos se le propone observar, analizar e interpretar su práctica durante la estancia de tres meses en un centro escolar, poniendo especial atención en algún aspecto relacionado con la didáctica de la lengua; se reflexiona sobre bibliografía relacionada con dicha metodología y se dan a conocer experiencias de anteriores alumnos en prácticas.

2. Las entrevistas. El corpus de investigación está constituido por segmentos de actividades típicas relacionadas con la enseñanza de las lenguas (Lengua Extranjera y Euskera). Dichas actividades se han llevado a cabo por alumnos de Grado en Magisterio de Educación Primaria durante el practicum del cuarto curso. Se ha trabajado con diez alumnos. Cada alumno ha seleccionado una secuencia de aproximadamente quince minutos de la actividad realizada en clase que tenga para ellos una relevancia especial (en total, hemos analizado 150 minutos de actividad real). A continuación, en un lapso de unos días, se han realizado entrevistas de confrontación a las imágenes de la actividad real. Cada entrevista ha durado alrededor de una hora (en total, diez horas de entrevista). En estas entrevistas se han implicado el formador-investigador y al actor.

En el transcurso de la entrevista de autoconfrontación, la formadora, de una manera sutil, sin ánimo de juzgar y sin que influya de manera prescriptiva, ha guiado al alumno para que este, al visualizar su propia actuación en la secuencia grabada en clase, la rememore y vuelva a experimentar lo sentido en aquel momento. En la autoconfrontación el formador-investigador pretende que el actor indague en la actividad dinámica que tiene ante sí. Para ello es necesaria una consigna clara que 
focalice la atención del actor en el mismo momento del aula para llevarlo hacia el análisis minucioso de lo sucedido. Así, el profesor novel podrá analizar y reflexionar sobre lo que le parece acertado y lo que se podría mejorar sin que la formadora se lo señale. "¿Qué es lo que estoy haciendo aquí? ¿Cuáles son mis miedos o mis problemas? ¿Qué me ha impedido llevar a cabo mi plan?...".

Esta interacción verbal entre actor e investigador no es una conversación habitual; en ella se pretende comprender lo sucedido en el aula desde el punto de vista del actor, incitado por la exposición a las trazas de su experiencia. La entrevista debe crear un entorno confortable para que el actor pueda sumergirse en su actividad y adoptar un "habla operante" (Merleau Ponty citado en Richir, 1988), donde se intenta poner en palabras algo que no se sabe con antelación. Desde este marco, la autoconfrontación se convierte en un instrumento formador mediante el cual el actor hace emerger su experiencia, explora el significado de sus acciones y el contraste entre las imágenes y sus proyectos. De esta manera se abren posibilidades para una transformación futura.

En el caso de Mondragon Unibertsitatea, el dispositivo metodológico se prolonga hasta realizar la autoconfrontación cruzada (Clot, Faïta, Fernandez y Scheller, 2001), en la que a la vista de las actividades realizadas por un compañero, los demás comparten impresiones, comparan, formulan preguntas, muestran las tensiones que aparecen en el vídeo, etc.

3. El análisis de las transcripciones de las entrevistas de autoconfrontación. Para analizar minuciosamente las transcripciones, se ha segmentado el discurso transcrito por unidades temáticas o de contenido (Sanchez \& Rosales, 2005). Dichos segmentos se han organizado en columnas paralelas, donde en una de ellas se señala la actividad real y el momento en que se ha realizado (por minutos y segundos) y en la otra lo verbalizado por el profesor novel al visionar la actividad señalada. Para ello, los segmentos correspondientes a las tipificaciones de la acción se han analizado atendiendo a los siguientes focos: tipificación progresiva de la acción, dinámica desarrollada en estos contextos formativos, formas de habla divergentes entre actor y formador-investigador, y por último, los miedos, obstáculos y vías de mejora que verbaliza el propio actor.

\section{Resultados}

Los análisis realizados sobre los materiales empíricos producidos en las situaciones efectivas de enseñanza y las confrontaciones a sus trazas por los actores conjuntamente con los formadores-investigadores han tenido como primer objetivo el de explorar las acciones típicas que efectivamente se llevan a cabo en las escuelas en relación con la enseñanza de las lenguas.

El foco sobre la investigación de la formación nos ha llevado, por otro lado, a observar la dinámica desarrollada en estos contextos formativos, es decir, aquellos procedimientos, operaciones y sucesos que ocurren en la experiencia formativa que recorre el actor. Se trata, pues, de comprender los resortes tanto de la actividad docente como de los proyectos de 
transformación que imagina el actor frente a sus imágenes. Todo ello con el fin de ponerlos al servicio de la mejora de nuestros entornos de formación.

\section{La tipificación verbal de la actividad propia}

El objetivo de este apartado es dar a conocer cómo en las autoconfrontaciones, los profesores que contemplan sus propias actuaciones en clase describen sus acciones al tiempo que reviven su experiencia, desde un ángulo distinto.

En el siguiente ejemplo se puede apreciar cómo el profesor novel describe su acción tipificando la acción progresivamente, configuración que va guiada por el significado que revisten sus actos sucesivos en este caso, en una sesión de lengua:

P: Eso es, yo al final estoy dictando esa pregunta

F: Sí

P: Dictando bueno/ tampoco quería dictar/ yo quería que entendieran lo que les preguntaba y que contestaran como quisieran/ al final quería que escribieran la pregunta como quisieran/ pero les ha costado

P: Aquí quería/ que consiguieran su objetivo y se ve que apuntan palabra por palabra lo que yo les digo y/ y a menudo me esfuerzo por hacerlo de dos formas distintas/ reformulando la pregunta

El profesor novel $(P)$ dice haber anclado la acción que muestra la grabación en lo que para él es una acción típica (dictar) explicando el contexto en el que recurre a ella.

En algunas ocasiones, a la hora de describir la actividad visionada, el profesor modifica la denominación de su actividad, ya sea como consecuencia de la propia dinámica discursiva ya como consecuencia de la interacción con el investigador (F). Durante una de las autoconfrontaciones, el profesor novel da cuenta de los objetivos de la sesión impartida. Al verbalizar los objetivos, va reformulándolos.

P: ... Pues tienen que adivinar un personaje misterioso

F: Tu aquí estás describiendo y ellos tienen que adivinar quién es el personaje

P: Quién es el personaje/ sí// bueno eso fue muy fácil/ adivinarlo (sonrisas)

F: ¿Cuál era el objetivo del ejercicio?

P: Sí, sí / en el fondo no/ al final el objetivo no era ese/ el objetivo al fin y al cabo era que ellos aprendieran los adjetivos / da igual adivinarlo o no/ bueno/ la cuestión es que ellos en el rincón de literatura siempre trabajan un personaje misterioso/ entonces, pues un poco/ hacen, pues / no sé qué le ha gustado / o, un poco como mezclado y no utilizan adjetivos / entonces pues un poco se les incita a utilizar adjetivos.

El hecho de tipificar una acción en contexto formativo le ha permitido al actor precisar de qué objetivo se trataba o bien reconfigurar la actividad realizada bajo un marco descriptivo diferente. Este proceso de recalificación, frecuente entre los noveles, lo designamos retipificación. 


\section{Identificación de los obstáculos, dificultades y mejoras}

El revivir la acción en un contexto de formación da pie a que el enseñante discurra sobre las dificultades, mejoras y posibles cambios en su forma de actuar y permite imaginar la actividad futura y configurarla. En la búsqueda de alternativas, además de recurrir a experiencias personales, recuerda las experiencias e intervenciones de otros profesores, lo aprendido a través de lecturas, etc. La autoconfrontación da opción a un juego de espejos donde el autor puede tomar distancia de su propia acción y analizarla como si fuera ajena, además de evocar una constelación de actividades (propias, ajenas, imaginadas). El análisis de la actividad que estos métodos pretenden favorecer toma en cuenta el conjunto de factores que afecta el desarrollo de una sesión en el aula, es decir que más allá de tomar al actor como agente aislado lo relaciona con un conjunto de factores que constituyen el medio escolar, tales como la metodología, las acciones de los alumnos, sus disposiciones, el ritmo y la duración de la tarea, entre otros. Vemos en el ejemplo cómo la auto-observación lleva al novel a considerar otras orientaciones metodológicas cómo alternativas preferibles.

P: Cada uno un tema distinto/ sí/ sí/ en ese sentido sí que hay que mejorarlo

F: ¿Mejorar en qué sentido?

P: Pues, escoger un tema sería más fácil para que ellos fueran capaces de relacionar y tener una visión más completa del tema

F: Te refieres a un proyecto

P: Sí/ trabajar mediante proyectos

Otras veces el enseñante ve la necesidad de transformar su intervención, como ocurre en el siguiente ejemplo:

P: Ahí compara/ bueno/ ahí igual le dije yo/ hubiera sido mejor, quizás que lo hubieran dicho ellos/ pues que se dieran cuenta con una comparación/ igual/ tiene el rabo tan suave como/ comparar un poco.

Como se aprecia en el ejemplo, la profesora identifica como un problema el hecho de que sea ella misma la que de los ejemplos y definiciones. Ante esta situación propone como mejora sustituir sus definiciones por otras estrategias -tales como facilitarles el percatarse de qué animal se trata mediante comparaciones- en las que el alumno sea el protagonista. Es frecuente que el novel al observar su trabajo apunte para su mejora el delegar la agentividad sobre el alumno reteniendo su tendencia a un excesivo protagonismo en la dinámica de la enseñanza. Este aspecto constituye un avance considerable en la evolución del novel hacia modalidades expertas de enseñanza.

También emerge la complejidad que supone para el profesor novel llevar a cabo las actividades diseñadas anteriormente:

P: Fui yo la que empezó a utilizar la estructura. /Empecé demasiado pronto. / Igual tenía que haber esperado.../Corrijo demasiado/ Tenía que haber esperado un poco más a ver si 
lo corregía él. / Las instrucciones deben ser muy claras, claras y pausadas... / Hay que esperar si alguien no está escuchando...

En definitiva, durante la visualización de su actividad se percatan de si el resultado responde o no a sus expectativas, y en la negativa, consiguen precisar de qué manera se podría transformar la actividad para su mejora. Estos resultados son muy beneficiosos para su propia formación como docentes.

\section{Tipificaciones en conflicto}

Ocurre en ocasiones que entre la profesora novel y la formadora no haya acuerdo en cuanto al tipo de actividad escolar que están visionando en la grabación. Este fenómeno calificado de forma de habla divergente (Plazaola y Ozaeta, 2014) ocurre cuando la inteligibilidad de la actividad escolar que están analizando, el modo de entender de qué actividad se trata, no coincide. En el ejemplo la formadora atribuye el tipo "escritura", mientras la profesora novel se resiste una y otra vez a que la actividad que ha realizado en clase se considere como tal.

P.: este día era un poco para contar lo que iban a hacer en la excursión...

$\cdots$

P.: fue aquí... ¿qué vamos a llevar para la excursión? ¿cuándo iremos? ¿a dónde iremos?

F.: es tu plan, ¿eso es lo que estás haciendo aquí?

P.: en realidad se trata de centrarlos en la excursión, o sea a dónde vamos... escribir una nota para que desde el principio sepan lo que van a hacer y luego todos los ejercicios ligarlos a eso. Por ejemplo, veremos animales, entonces... juegos con animales; o bien la huerta, visitaremos una huerta...

F.: es decir, que se trata de ver el plan con antelación...

P.: sí

F.: y escribes el plan

P.: para que después ellos lo escriban en su hoja, algunos pueden copiarlo desde aquí o si no a medida que lo digo, que lo escriban a su manera.

$\cdots$

P.: el objetivo no era aprender cómo se escribe sino más bien que supieran lo que íbamos a hacer

P: Sí, de hecho, ellos luego lo tienen que copiar, así que... (cambiando el tono, como si los niños preguntaran enfadados) ¿dónde empieza? ¿qué...?

F: ¿Para qué se lo hacías copiar, para leerlo?

P: Para que se dieran cuenta de qué íbamos a hacer, luego tenían que escribirlo. 
P: Aquí les estoy diciendo que escriban la nota. La escribirán en papel de colores y luego, con eso harán un dibujo para llevárselo a los padres, sobre todo para que sepan qué harán (al día siguiente).

F: Se trata de copiar en una parte.

P: No. Les digo: si queréis, copiad por aquí y si no, hacedlo vosotros a vuestra manera. El que pueda, como pueda. No tienen todos el mismo nivel para escribir.

....

F: Sí, yo creo que ahí... este, por ejemplo, ha copiado todo bien, bueno bien... (tono de duda), parece que ha seguido la secuencia: botas, katiuskas, chubasquero.

P: Quizá la copia ha sido más fácil.

F: ¿Qué plan tenías ahí? (la formadora duda al formular la pregunta)

P: El objetivo no era que aprendieran cómo se escribe, sino que ellos supieran qué iban a hacer.

F: ¿Pero ¿cómo lo sabrán si no lo entienden?

P: Porque lo hemos dicho, es decir, porque lo hemos planificado de antemano, porque hemos decidido entre todos qué es lo que verían más o menos. (Los movimientos de sus manos evidencian cierto nerviosismo ante las preguntas de la formadora)

F: Quizá habría que atender a los objetivos de escritura.

P: Quizá no tuve tanto en cuenta eso, y no fue escritura sino copia.

La cuestión de definir, de tipificar de qué actividad se trata vuelve una y otra vez. La profesora se resiste a la propuesta de la formadora, que ve en el desarrollo de la actividad escolar una situación propicia para la lectoescritura. La última intervención de estos extractos muestra claramente que para la profesora novel "no fue escritura, sino copia".

Estos análisis nos muestran que, en formación, cuando se trabaja ante grabaciones de los futuros profesores, la cuestión de la tipificación de la actividad observada es fundamental y no debe darse como evidente y consensuada. Se trata en realidad de un momento clave de la formación en el cual es importante que el formador atienda a la manera en que el actor tipifica su propia acción, qué es según él lo que ha realizado en clase.

\section{Conclusiones}

En esta presentación hemos subrayado los aspectos teóricos sobre la actividad humana que fundamentan el proyecto de formación-investigación en formación inicial del profesorado que llevamos a cabo.

La concepción de la actividad humana en la que nos apoyamos toma en cuenta las reservas de experiencia del actor, es decir sus experiencias anteriores, como punto de partida de la profesionalización que se trata de desarrollar. Para ello es necesario tomar en cuenta el significado que la actividad realizada reviste para el actor, lo cual se manifiesta en la manera en 
la que el actor tipifica verbalmente la actividad realizada. El entorno formativo toma especial precaución en el modo (no prescriptivo) de acompañamiento de las entrevistas, lo que induce al profesor novel a pasar del análisis de la actividad realizada hacia las modificaciones que supondrían su mejora. Los resultados muestran estas transformaciones virtuales, lo que ha permitido fundamentar dispositivos de formación tanto de materias del curso como del seguimiento del Practicum y de trabajos de fin de grado. Se busca así la mutua fecundación entre formación e investigación sobre la formación.

El dispositivo metodológico utilizado, asimismo, puede permitir indagar en la cultura de los centros educativos a través de la diversidad de las actividades de enseñanza, sus configuraciones y significados diversos en función de actores y culturas, y su dimensión colectiva e institucional. Para ello, no obstante, será imprescindible ampliar el corpus introduciendo el análisis de la actividad real de los profesores experimentados y profundizar en los retos metodológicos y prácticos que pueda plantear el análisis.

Cabe señalar que el proceso formativo que experimentan los alumnos no finaliza, necesariamente, en las entrevistas de autoconfrontación, puesto que en el Trabajo de Fin de Grado varios alumnos optan por reflexionar y profundizar en aspectos especialmente relacionados con la didáctica de la lengua, tanto desde su práctica como en relación a la teoría que la sustenta.

Por último, quisiéramos señalar que estas experiencias muestran que el dispositivo metodológico empleado incide en el futuro profesor y le ayuda a aproximarse al oficio y le sirve para conocer los obstáculos comunes de los profesores noveles. Asimismo, el experimentar la formación por medio de estas técnicas supone una iniciación a nuevas maneras de formarse que podrán incidir en su desarrollo profesional a lo largo de la vida.

\section{Bibliografía}

Alsina, A. (2013). Un modelo realista para el desarrollo profesional en la formación inicial de maestros de educación infantil. Revista Electrónica Interuniversitaria de Formación del Profesorado, 16 (2), 27-37. Disponible en http://dx.doi.org/10.6018/reifop.16.2.180761

Arocena, E., y Gorter, D. (2013). The multilingual classroom in primary education in the Basque Country and Friesland: beliefs of teachers and their language practices. Mercator European Research Centre on Multilingualism and Language Learning. Disponible en http://www.mercatorresearch.eus/fileadmin/mercator/publications_pdf/MultilingualClassro omWEB.pdf

Bajtín, M. (1982). Estética de la creación verbal. Madrid: Siglo XXI editores.

Blin, T. (1999). Phénoménologie de l'action sociale: A partir d'Alfred Schütz. Paris: L'Harmattan.

Bronckart, J.-P. (1997). Activité langagière, textes et discours. Pour un interactionnisme sociodiscursif. Paris: Delachaux et Niestlé. 
Cefaï, D. (1994). Type, typicalité, typification: La perspective phénoménologique. En B. Fradin, L. Quéré y J. Widmer (Eds.), L'enquête sur les catégories: De Durkheim à Sacks. (pp. 105-128). Paris: Editions HESS.

Clot, Y., Faïta, D., Fernandez, G. y Scheller, L. (2001). Entretiens en autoconfrontation croisée: Une méthode en clinique de l'activité. Éducation Permanente, 146 (1), 17-25.

Dewey, J. (1933). How we think (rev.ed.). Boston: Heath.

Durand, M. (2008). Un programme de recherche technologique en formation des adultes. Éducation et Didactique, 2(3), 97-121.

Gaudin, C. y Chaliès, S. (2012). L'utilisation de la vidéo dans la formation professionnelle des enseignants novices: revue de littérature et zones potentielles d'étude. Revue Française de Pédagogie, 178, 115-130.

Hatch, T., y Grossman, P. (2009). Learning to look beyond the boundaries of representation. Journal of Teacher Education, 60(1), 70-85

Leblanc, S., y Ria, L. (2014). Designing the Néopass@ction Platform based on Modeling Beginning Teachers' Activity. Design and Technology Education: An International Journal, 19(2).

Lussi Borer, V., Muller, A., Ria, L., Saussez, F. y Vidal-Gomel, Ch. (2014). Conception d'environnements de formation: Une entrée par l'analyse de l'activité. Activités, 11(2), 72-75. Disponible en http://www.activites.org/v11n2/Ria.pdf

Mir, M.L. y Ferrer, M. (2014). Aproximación a la situación actual de la formación del profesorado de educación infantil. Revista Electrónica Interuniversitaria de Formación del Profesorado, 17 (2), 235-255. Disponible en http://dx.doi.org/10.6018/reifop.17.2.181441

Montmollin, M. (de). (1996), L’Ergonomie. Paris : La Découverte.

Plazaola Giger, I. y Ozaeta, A. (2014). Les formes de la parole dans l'entretien formatif d'autoconfrontation. Activités, 11(2), 112-128. Disponible en http://activites.org/v11n2/Plazaola.pdf

Quéré, L. (1994). Sociologie et sémantique: Le langage dans l'organisation sociale de l'expérience. Sociétés Contemporaines, 18-19, 17-41.

Ria, L. y Leblanc, S. (2011). Conception de la plateforme de formation Néopass@ction à partir d'un observatoire de l'activité des enseignants débutants: Enjeux et processus. Activités, 8 (2), 150-172.

Richir, M. (1988). Relire la « Kriss» de Husserl. Pour une position nouvelle de quelques problèmes phénoménologiques fondamentaux, Esprit, 7 - 8, 129-151.

Rosch, E. (1978). Principles of Categorization. En E. Rosch y B.B. Lloyd (Eds.), Cognition and Categorization, (pp. 27-48). Hillsdale, New Jersey: L. Erlbaum.

Salembier, P., Theureau, J., Zouinar, M., \& Vermersh, P. (2001). Action/Cognition située et assistance à la coopération. En J. Charlet (Ed.), Ingénierie des connaissances IC2001 (pp. 369388). Grenoble: PUG. 
Sáchez, E., y Rosales, J. (2005). La práctica educativa. Una revisión a partir del estudio de la interacción profesor-alumnos en el aula. Cultura y Educación, 17 (2), 147-173.

Schütz, A. (1993). La construcción significativa del mundo social. Introducción a la sociología comprensiva. Barcelona: Paidós.

Seidel, T., Stürmer, K., Blomberg, G., Kobarg, M., y Schwindt, K. (2011). Teacher learning from analysis of videotaped classroom situations: Does it make a difference whether teachers observe their own teaching or that of others?. Teaching and teacher education, 27(2), 259267

Suchman, L. (1987). Plans and situated actions. The problem of human-machine communication. Cambridge: Cambridge University Press.

Theureau, J. (2010). Les entretiens d'autoconfrontation et de remise en situation par les traces matérielles et le programme de recherche "cours d'action". Revue d'Anthropologie des Connaissances, 4 (2), 287-322.

Voloshinov, N. (1992). El marxismo y la filosofía del lenguaje. Madrid : Alianza. 